\title{
ViNET Infrastructure Model for Internet Connectivity in the Scenario of Dhaka City
}

\author{
Md Nakibul Alam \\ Department of EECE \\ Military Institute of Science \\ and Technology
}

\author{
Md Abdullah Al Faruk \\ Department of Computer \\ Science \\ American International \\ University - Bangladesh
}

\author{
Rifat Tasnim Anannya \\ Department of Computer \\ Science \\ American International \\ University - Bangladesh
}

\author{
D.M. Shafin Ahmed \\ Department of Computer \\ Science \\ American International \\ University - Bangladesh
}

\begin{abstract}
Today's world is now rapidly moving towards ubiquitous connectivity. Every other device is getting connectivity among each other. While forming a network among themselves, these devices are being used by users so that their lives can be easier than ever. Bangladesh is a country containing more people than its capability. With the technological encroachment in the world, as a developing country Bangladesh is also trying make sure that all its citizens get the right to access information, one of the assets considered today. Today access to information has become a basic right to everyone. To ensure peoples' access to internet is really important. Currently the residents of the country are using mediums and connectivity like broadband, WiMAX, and cellular networks to access the internet. A lack of centralized government provided internet infrastructure can be observed. People here also spend most of their times on roads, stuck in a traffic jam. In this paper an approach to create a new internet providing structure, ViNET, Vehicular Internet, in the context of Dhaka city is introduced where, the concept of VANET and a few existing technologies is been used. With the help of this infrastructure model, people will be able to get connectivity even when they are on the roads, stuck in traffic jam and passing a road. By implementing this infrastructure access to information will be ensured. Also, the traffic problem can be utilized in a better way to make it also useful. Another useful part of this infrastructure will be the reduction on the dependency from the cellular network providers. Also, many more social services as well as emergency services can be provided by the government. By using this infrastructure peoples' right to access to information can be achieved. In this paper, the infrastructure elements are described, the technologies are also discussed which are been used in it. Also, simulations are run using the processes been held in this infrastructure to show that it will be able to provide enough connectivity to end users.
\end{abstract}

\section{General Terms}

\section{VANET}

\section{Keywords}

Network infrastructure, internetworking, Vehicle based network infrastructure.

\section{INTRODUCTION}

Bangladesh is a small country holding the population more of its capacity. The living residents of the capital Dhaka city is 14.4 million which is far more than the land is capable of holding. The main transportation of these huge amount of people is by road while going to their destinations. To carry such amount of people a lot of vehicles such as - bus, cars, micro buses, CNG auto rickshaw, bike etc. are been used. This big number of transportations or vehicles contain most of the streets and roads and keep them busy almost all day long.

According to a report of World Bank published in the year of 2017, it has been reported that, the average traffic speed in Dhaka city has reduced to $7 \mathrm{kmph}$ from $21 \mathrm{kmph}$ since the last few years. It can be said that, this slow traffic speed puts an adverse impact on the socio-economic advancement of the entire country as Dhaka is the capital of the country. To reach a small distance it takes a lot of time than it should require. It reduces the efficiency of the entire city. Traffic congestion is one of the main problems of the city alongside the crisis of gas, water and electricity. Despite of these challenges the country is moving towards the global connectivity.

Today's world is somehow completely dependent on information and communication technology. Information is considered as an asset today. Access to information has become kin of a basic right for human beings all around the world. For this to happen, Internet plays a very crucial role. It is considered as an essential part of our life. In underdeveloped and developing countries like Bangladesh, Internet is now playing a crucial role in the socio-economic advancement of the country.

World is now moving towards the ubiquitous connectivity. Every other device is connected with each other. Concepts like IoT or IoE are growing and taking attention of people very rapidly. Same is happening in our country too. That time is not so far when in our country concepts like IoT or IoE are going to be implemented. For implementing those concepts, Internet of Things or Internet of Everything, connectivity among everything is required. Also, all mobile devices are getting connectivity now a days. For that seamless internet connectivity is needed. In our country as described previously, internet connectivity is not covering entirely. The mediums are limited. The sources are limited. Also, a huge lack of infrastructure is observed.

In this paper a new infrastructure model using the concept of internet infrastructure is proposed as well as proof has been provided that it can provide connectivity to a lot of people in the urban scenario of Dhaka city.

Popular research concern such as, VANET and some existing cost-effective technologies in the perspective of Bangladesh is used in the described model. The model is proposed to provide connectivity to people on the roads utilizing one of the greatest problems of the country, high density of traffic. Using this infrastructure people of the Dhaka city can utilize their time while stuck in traffic jam. This will ensure peoples' access to internet and information to full fledge. Internet of 
Things or Internet of everything can be implemented by this seamless internet connectivity. Cost of uses of internet can also be reduced. Simulations are also run in Arena Simulation tool, where it is found that by the processes run by the proposed model will be able to provide connectivity to a large number of people while making the best use of the huge number of vehicles on the road. In this structure the concept of VANET or Vehicular Ad-hoc Network is used. VANET is a very hot topic for researchers around the globe for the last few years. In modern world countries VANET is used to provide numerous safety and non-safety applications to users while moving on roads in their vehicles. That concept will be absorbed in the context of Dhaka city and it is named as ViNET or Vehicular Internet, where the vehicles will be considered as an access point that will broadcast a single SSID in the entire city and provide connectivity to people on the roads. Also, fiber optic communication will be used so that the back bone of infrastructure remains seamless. Wireless access points with wireless distribution system capability will be used where, these access points will work both as a wireless extender and access points to cover up the entire Dhaka city area. The concept of VANET of will be used but the hardware been used in VANET will not be used as the vehicles in our country are not capable to carry that hardware and that hardware are rare in our country. But the access points are been used broadly in Bangladesh.

\subsection{Problem Definition}

Currently in Bangladesh, and in the its capital Dhaka city, people are getting Internet connectivity via broadband, WiMAX, 3G/4G cellular networks. For this reason, people are completely dependent on these service providers. A centralized government provided internet facility is a huge lack in our country. People has to pay a huge amount of money These are used in office or home environments. Cellular network is utilized in public environments also. For public environment, a few Wi-Fi spots can be found, typically at restaurants, universities etc. For which while moving on the roads people need to completely rely upon cellular networks. Besides that, the motivation of this research work is to find a new way to build a new infrastructure for providing flawless internet connectivity by utilizing one of our city problems in a better way which the traffic.

\subsection{Aim}

This thesis is all about providing a infrastructure model of a new Internet infrastructure model in the context of Bangladesh, while using the concept of VANET, technologies like - optical fiber, repeater, extender, Wi-Fi, WDS and WMN etc. which are enough possible to implement in the present scenario of the country. It is also shown through simulation that, the huge number of vehicles on the roads, which will work as AP, will be more than enough to provide client association. At the back-end AP controllers will be used at the central data center, from all the communications will be maintained. Root access points will be used to maintain the backbone connectivity between the data center and all other access points.

\subsection{Scope}

The scope of this research does not include the security issues, cost efficiency as well as backend infrastructure. In general, it will be considered that at the back end, data center will be responsible for maintaining the backbone connectivity of the entire network.

\section{LITERATURE REVIEW}

The main infrastructure is designed following the concept and architecture of VANET. In [1], authors have described about the physical architectures of VANET In VANET (Vehicular Ad Hoc Network) as infrastructure is used -

\section{- RSU: Road Side Units}

- Used for making the communication of the vehicles with internet

- Uses Wireless $802.11 \mathrm{a} / \mathrm{b} / \mathrm{g}$ for communication

- It is connected with the centralized servers via internet with the help of cellular network

\section{- OBU: On Board Unit}

- Contains GPS, Accelerometer, Wi-Fi device, DSRC device

\section{- Gateway}

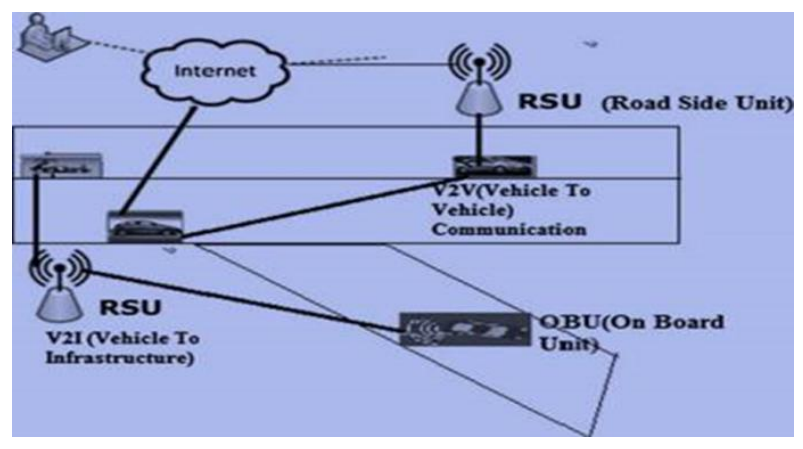

Fig 1: VANET Architecture

Wireless networks are convenient, cost effective and easy to integrate with all other network and network component without any extra technical hitches. For this reason, it is easy to setup and maintain. In [Designing of Wireless Distribution System Network (WDSN) Model], authors have developed a WDSN or wireless distribution system network using WDS in order to bridge WLANs or wireless local area networks. They have run web browsing, email, audio streaming, and FTP. They have also run simulation in OPNET.

In [2], the capability of WDS technology to work both as a repeater and extender along with client association is described.

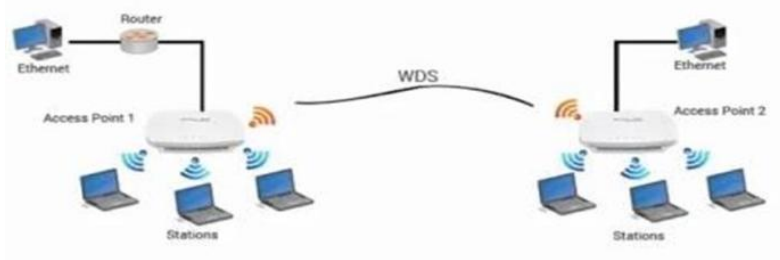

Fig 2: Wireless Distribution System

In [3], wireless mesh networks feasibility is provided. Wireless Mesh Network (WMN) is a communication network made up of radio nodes organized in a mesh topology. It is also a form of ad hoc network. It consists of mesh routers, gateways and mesh clients. 
WMN is less expensive than traditional network as all the nodes are connected with each other. It is tremendously acceptable, adaptable and expandable. It can support high demand.

In [4], Cisco, one of the largest networking technology providers has described about their products for wireless mesh Access Points

In [5], Cisco has described about their product's deployment in wireless mesh network

In [6], [7], has described about their products for wireless mesh access points where a root AP will broadcast the SSID and leaf APs will capture that signal of the root and extend to other Apps. They will also do the client association

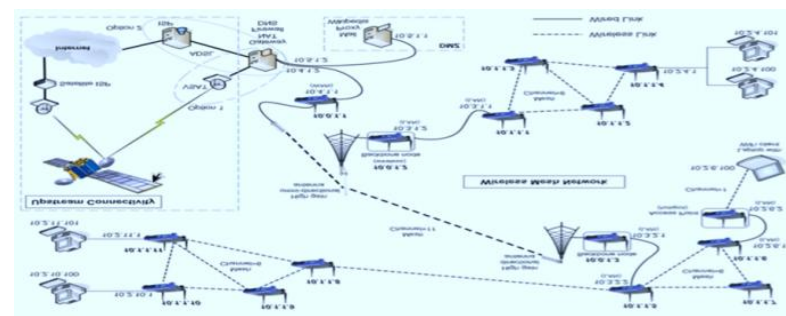

Fig 3: Wireless Mesh Network Architecture

\section{RELATED TECHNOLOGIES AND CONCEPTS}

\subsection{Wireless Distribution System (WDS)}

Wireless networking has become an essential part as well as a well-acknowledged standard in every area, such as- home, small office, business etc. The motive behind is it is cheaper and convenient than traditional wired networking infrastructure. The difficulty to cover a large area wirelessly, or to extend a wireless network in a large area is one of the problems in wireless network. A technology named WDS was introduced to overcome this challenge in 2005-2006 [9]. WDS means Wireless Distribution System.

In WDS technology multiple wireless devices are put up in bridge mode which empowers them to get connected with each other devoid of the help of wires.

A WDS permits access points to converse with each other without the help of any wires but in a standardized way [10].

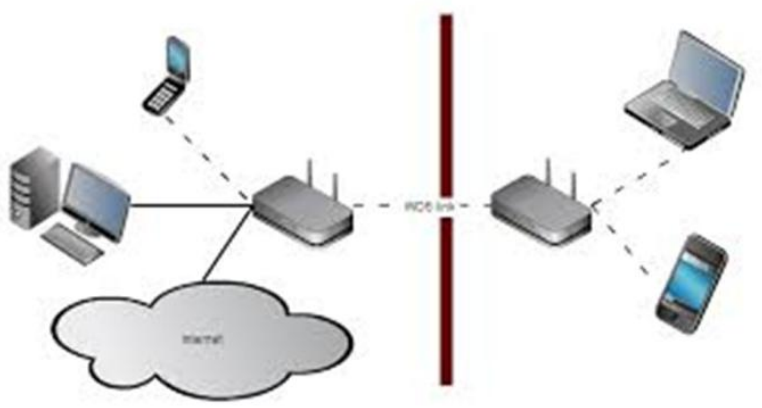

Fig 4: Wireless Distribution System

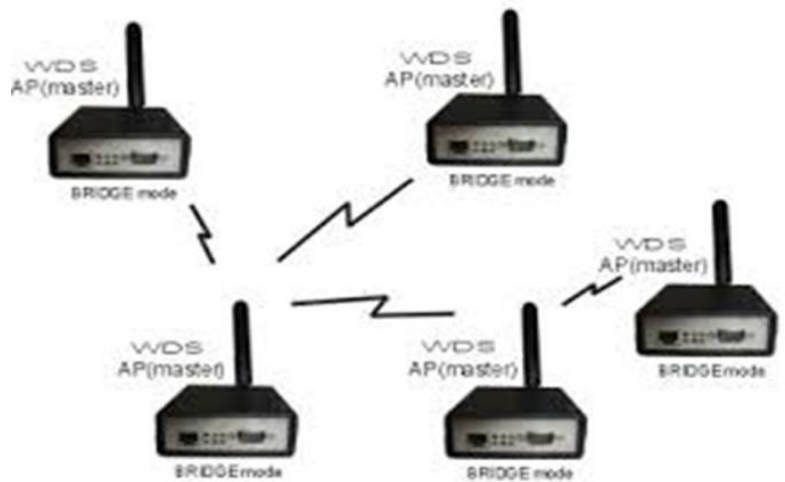

Fig 5: Wireless Distribution System (Master - Bridge)

The prerequisite of WDS is the use of MAC or Media Access Control address to launch a link among WDS group. Its origins the connectivity to the Data Link layer which is just below the Network layer of OSI model.

This feature WDS of Access Points facilitates users a seamless connectivity which makes managing, multiple wireless networks easier. It also reduces the overweight number of cables to connect those networks.

The Wireless Access Point (WAP) can work as a single $\mathrm{p} 2 \mathrm{p}$ or point-to-point access point, $\mathrm{p} 2 \mathrm{mp}$ or point-to-multipoint bridge or as a repeater.

\subsubsection{Point-to-Point Access Point}

For Point-to-Point Access Point, a single wireless access point can accept connectivity from users and other devices in the network.

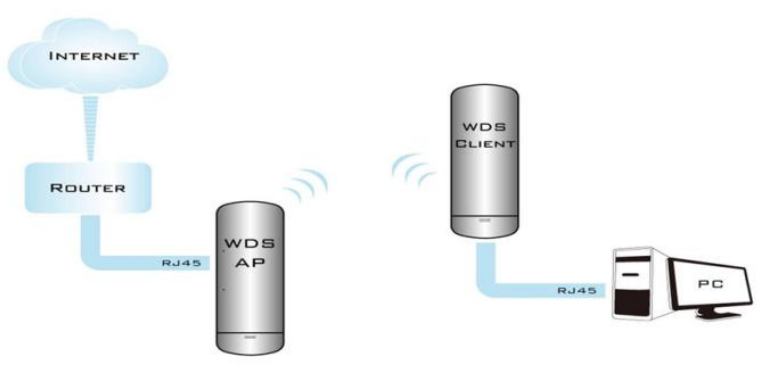

Fig 6: Point-to-Point Access Point

\subsubsection{Point-to-Multipoint Bridge:}

For Point-to-Multipoint Access Point, a single wireless access point can act as a conjoint medium of communication among multiple access points

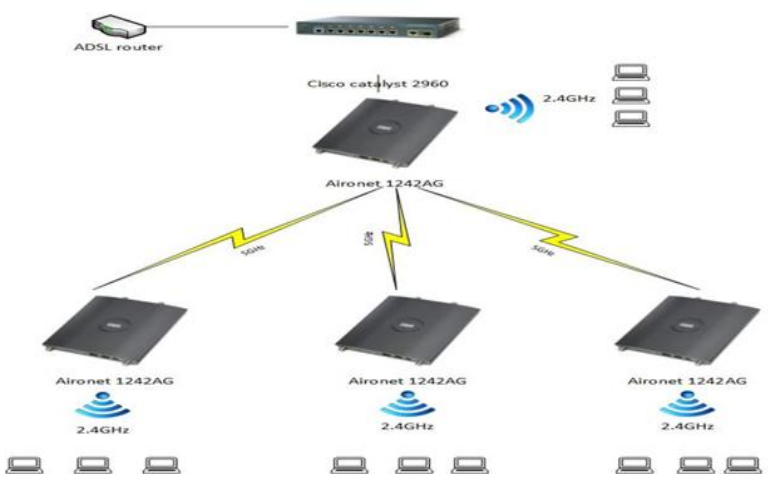

Fig 7: Point-to-Multipoint Bridge [8] 


\subsubsection{Repeater Mode}

For Repeater Mode, an access point can perform a connection formation among wireless access points which are far away from one another.

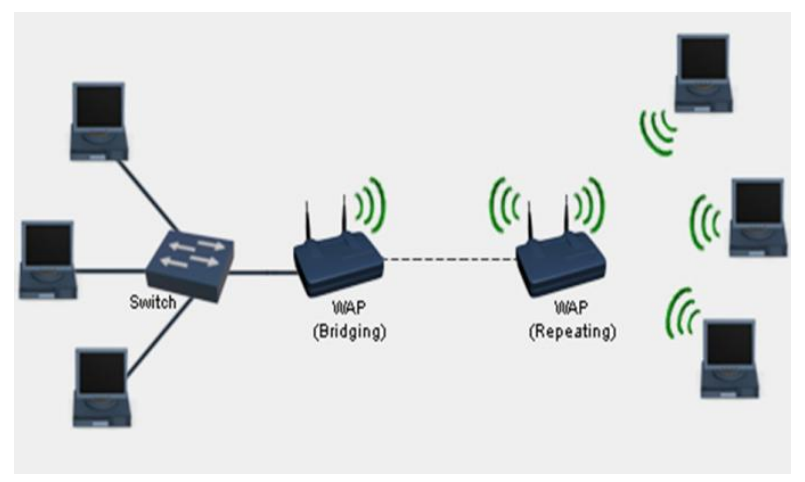

Fig 8: Repeater Mode Access Point

It needs to be mentioned that, users can get connected with these repeaters. For which a WDS role is equivalent to the role of a repeater.

\subsection{Repeater}

To enhance coverage by amplifying and re-transmitting mobile signals to improve coverage in areas where it is required or poor mobile repeaters, which are wireless devices are used. Mobile reportage can be heightened by use of a repeater on a local level. But it often causes interference to other users by doing so in an ad hoc way, particularly if the repeaters operate outside the direct control of the mobile operator.

Not only for voice and text but for many other applications, the propagation of device types and the trundle out of ever faster mobile technologies has made their use rife, whilst mobile coverage has always been incomplete, expectations have risen and dissatisfaction with 'not-spots' has grown. As mobile devices are been utilized every now and then so the impact of gaps in reportage are felt more sternly by the user. There are sound technical reasons for which the coverage delivered by the focal network will not penetrate certain areas. Metallization of windows, the walls of buildings for thermal reasons, and the use of underground spaces all diminish the signals between mobile and base station. This attenuation of the signal will tip to a reduction of data rate in $3 \mathrm{G}$ and $4 \mathrm{G}$ systems, and in due course to a loss of connection in all systems. There are some possible solutions. They include pico-cell base stations and repeaters.

A Pico-cell is a trivial, low power version of an orthodox mobile phone base station to provide fill-in coverage in a limited area. A repeater elite up a signal from the base station, amplifies and re-transmits it in the area of meager coverage; similarly, it amplifies and re-transmits the signal from a mobile in the exaggerated area to the base station.

\subsubsection{Interference Problem}

The mobile networks are carefully planned to maximize coverage and capacity with a finite amount of radio spectrum. This involves plotting the signal strength from each antenna of each base station in detail and coordinating frequencies (or scrambling codes for $3 \mathrm{G}$ ) between base stations. The network operators install repeaters themselves, or via approved contractors, to enhance coverage in some areas.

They avoid interference problems by planning the signal levels and frequencies to fit in with their wider network. The repeaters will also usually operate on one network only. The types of self-installed repeaters operated in the UK typically take the whole of a mobile band, amplify and re-transmit it. Signal strengths and noise levels across the whole band will be increased, meaning that all networks operating in that band are affected. In addition, the signal levels are not planned in the way that they are with operator-installed repeaters. Fixing a coverage problem for a small number of users can result in degrading coverage for a much larger number of users.

There are several different mechanisms by which a repeater could interfere with the operation and performance of the mobile network. Such as -

- Raising the noise floor on the uplink signal

- Raising the noise floor on the downlink signal

- Blocking (overloading) the base station receiver

- Disrupting the uplink power control

- Oscillations and spurious emissions

- Distortion or delay of the signals

Some of these are not significant in practice for various reasons, all are described in more detail below:

\subsubsection{Raising The Noise Floor - Uplink}

The repeater will transmit not only the wanted signal but wideband noise. Because any electronic device is thermal noise inherent in its components. The combination of the amplifier gains and noise figure means that the noise floor at the output could plausibly be $80 \mathrm{~dB}$ above thermal noise. In addition to any noise at the input from external sources will be amplified along with the wanted signal. If there is a relatively small path loss from the repeater to the base station then the noise transmitted by the repeater can be above the sensitivity level of the base station's receiver. In this case the base station's effective sensitivity is reduced. For mobiles at the edge of the cell, already operating at the upper end of their power control range, the uplink connection will be lost. The user will see the symptom as a dropped or blocked call even if their mobile shows there is network coverage. The mobile is at the cell edge so the interference mostly has impact mobiles that are some distance away from the repeater. The operator will see a worse than expected signal to noise ratio (SNR) in their network monitoring system. In $3 \mathrm{G}$ and $4 \mathrm{G}$ systems the data rates are variable for each link and in total. Increasing the noise floor reduces the SNR. This causes an increase in bit errors that in turn will cause the radio link control to increase the mobile's power. If it is not possible to increase the mobile's power, either because it is at maximum already or for load balancing reasons, then the data rate will be reduced. The effect of the repeater noise is therefore to reduce the capacity of the cell even if the connection is not dropped. This will affect all users in the cell.

\subsubsection{Other Network Interference}

When the user is in a poor coverage area for their network operator, this situation is most likely to occur, but a good coverage area for another operator. It is the second operator's base station that is interfered with. Thus, the affected mobiles are not only distant from the repeater but on a different network to the repeater user.

Repeaters that only operate on a single network avoid this interference mechanism. In addition, any repeaters installed by the MNO can be planned to have locations, power levels, antenna patterns etc. that avoid the problem. 


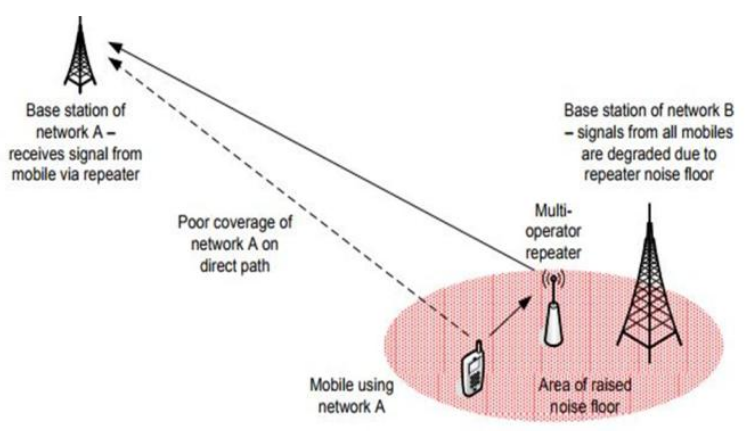

Fig 9: The Near-Far Problem in The Uplink

\subsubsection{Same Network Interference}

This situation occurs where the outdoor coverage is good but there is a high penetration loss to the user's location. This may be at the center of a large building such as an office block or hotel with many rooms; it may be the basement floors of a shopping center or multistorey car park; or it may be inside a vehicle or building that is largely metallized for reasons of thermal insulation. In these cases, the wanted base station may be quite close to the repeater, and so is affected by the raised noise floor. This reduces the coverage area for other mobiles that are on the same cell but outside the repeater's coverage.

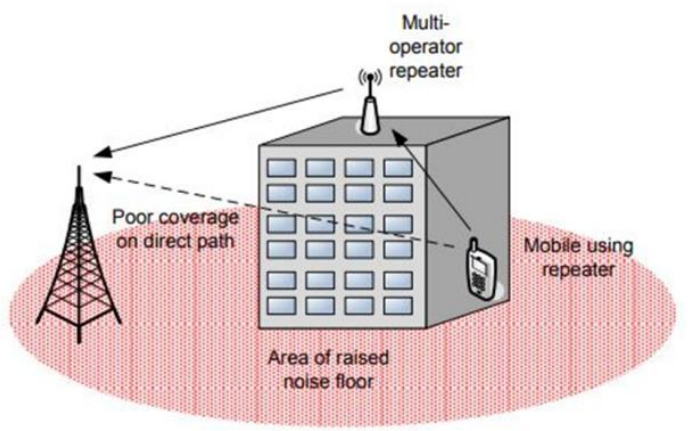

Fig 10: The Low Path Loss Problem

Since the path loss between repeater and base station is relatively low, the gain can be reduced and with it the noise floor. This requires some degree of automatic gain control in the repeater's uplink, based on the signal level received in the downlink. For a multi-operator repeater to be effective both in preventing interference and as a repeater, the gain control will need to act separately for each operator's spectrum. Cascaded repeaters are a particular problem. If repeater $\mathrm{A}$ is used to provide coverage to repeater $\mathrm{B}$, then on the uplink the noise out of repeater B is further amplified and added to by repeater A. This can create a much higher noise level than one repeater alone.

\subsubsection{Raising The Noise Floor - Downlink}

The mechanism for raising the noise floor in the downlink is similar in nature to that in the uplink, except that it is the mobile's receiver that is affected. As the repeater's downlink output power is a lot less than a macro base station, it is only mobiles near to the repeater that will be affected. The wanted output signal is amplified from a weak level to a much stronger level, that being the purpose of the repeater. For a multi-operator repeater, the noise level is raised for all mobiles in the vicinity, but so is the wanted signal. A mobile that is currently receiving a good signal may suffer a reduction in SNR. However, there is unlikely to be a significant interference problem from this effect. Mobiles that are currently receiving a weak signal are likely to benefit more from the increase in wanted signal than they suffer from the increase in noise - this is the purpose of the repeater.

\subsubsection{Base Station Receiver Blocking}

The repeater will transmit the signal from the mobile after amplifying it. If the repeater is near to a base station and at full gain then the signal at the input of the base station's receiver will be at a high level. If the level is too high then the receiver will be overloaded and it the reception of other mobiles will be degraded or stopped entirely. This process is known as blocking. In practice GSM, UMTS and LTE systems all use uplink transmitter power control (ULPC). This is particularly important in UMTS due to the use of code division multiple access (CDMA). The base station measures the signal level that it is receiving from a mobile and continuously instructs the mobile to increase or decrease its transmitter power. This maintains the signal level at the base station at its optimum level. An increase in the path loss to the mobile will result in the mobile being instructed to raise its power, until it is at maximum power. Any further increase in path loss will cause the mobile to go out of coverage. Area of raised noise floor multi-operator repeater Poor coverage on direct path Mobile using repeater 12 If the repeater gain is fixed then the ULPC function still works correctly. In this case the ULPC will adjust the mobile's transmit power as required and the repeater will not cause blocking.

\subsubsection{Power Control}

If changes in the mobile's transmit power are not carried through the repeater, then the ULPC breaks down, causing blocking as described above.

There are two apparent routes by which this may happen:

- The repeater is at its maximum power level. This has the same effect as the mobile reaching its maximum power - the mobile goes out of coverage

- The repeater employs gain control that maintains a steady output power on the uplink.

This will defeat the ULPC and is likely to reduce the capacity and/or coverage of the cell 3G systems are particularly sensitive to a failure of ULPC. If a mobile's power is too low it will be dropped; if it is too high it will reduce the capacity of the cell for other mobiles. The potential for interference to the cell therefore depends on the gain control, if any, that is used. Maintaining a constant gain is not a problem, maintaining a constant output power is likely to cause interference.

\subsubsection{Oscillation and Spurious Emissions}

At its simplest a repeater consists of two amplifiers, one for the uplink and one for the downlink. Diplexers are fitted to allow the output of one amplifier and the input of the other to use the same antenna. The main signal path for the downlink is from the base station to one antenna, through the downlink amplifier, and from the second antenna to the mobile. The reverse path applies to the uplink. However there exists another path that is unintended - the path between the two antennas. This acts as a feedback path from the output to the input of each amplifier. 


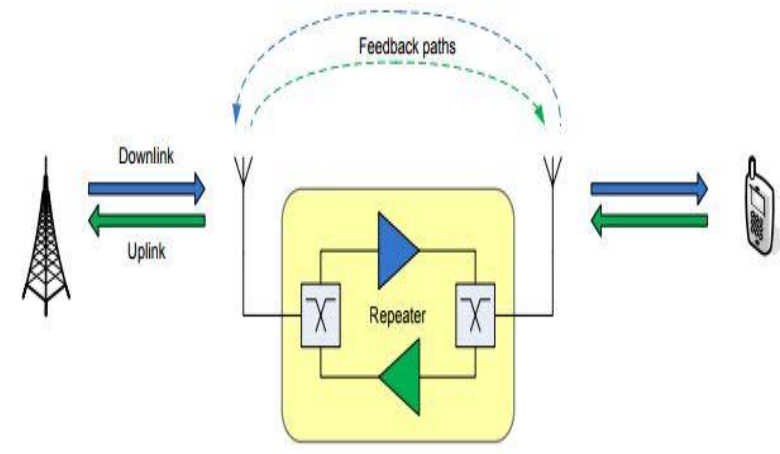

Fig 11: Feedback and Paths Around a Repeater

If the loss on the feedback path is less than the gain through the repeater then it will oscillate. The oscillation will occur in the operating frequency band and will be at the repeater's saturated output power. The effect on a base station or mobile using the oscillation frequency is that the repeater will act as a jamming transmitter. Many of the available repeaters have gains of over $50 \mathrm{~dB}$, which is a plausible path loss between antennas that are within a few meters of each other. For this reason, some repeaters include anti-oscillation features. Details are not disclosed but it is believed that these detect the feedback condition and reduce the gain as required to maintain stability. A similar effect is that of spurious emissions. These are signals that are generated internally within the repeater from various sources. They could be at any frequency but those within the operating band are likely to be amplified and so cause greater levels of interference.

\subsubsection{Distortion and Delay}

The repeater will not only amplify the wanted signals, in any real system it will also delay and distort them to some extent. The receivers in the mobile and base station can tolerate a certain amount of distortion without a problem, but excessive distortion will cause bit errors leading to a lowering of quality of service. The effects will be the same as for high noise levels: dropped or blocked calls, and reduced data rates. This will occur even when the receiver is picking up a strong signal. The distortion in the repeater is usually greatest when the signal level is high.

Another symptom of distortion is power being spread into the channels immediately above and below the main signal - the adjacent channels. This will raise the noise level in the adjacent channels and so increase the likelihood of interference to any base stations or mobiles operating on those channels. The delay through the repeater is mostly due to the filtering and signal processing rather than the amplifiers themselves. There is an inherent delay in both downlink and uplink paths anyway due to the speed of propagation and the distance between the mobile and base station. A distance of $300 \mathrm{~m}$ gives a delay of $1 \mu \mathrm{s}$. A cellular mobile system is designed to cater for variable delays in the signal path as the mobile moves around.

Different standards have different limits on round trip time (the time for the radio signal to travel from base station to mobile and back again) but they equate to a maximum range that is usually tens of $\mathrm{km}$. Adding delay in a repeater will take up part of this maximum round trip time and so reduce the maximum range. For most simple repeaters the delay is specified at $\leq 0.5 \mu$ s one-way. This means it reduces the cell radius limit by no more than $150 \mathrm{~m}$. In practice the cell is nearly always limited by path loss to a lower radius than the timing limit and a reduction of $150 \mathrm{~m}$ is unlikely to change this.

The delay in the repeater is therefore unlikely to have an effect on cell coverage. Smart repeaters may have longer delayed due to the signal processing and more demanding filtering involved. A $5 \mu$ s one-way delay will reduce the cell's maximum range by $1.5 \mathrm{~km}$. Unlike the noise problem, this range reduction affects only those mobiles using the repeater. Those operating directly with the base station are unaffected.

\subsubsection{In-Vehicle Repeaters}

Repeaters are available for installation on board a car, bus, train or boat. The lack of cellular coverage on trains has been described in a previous report by PA for Ofcom1. The key point is that each repeater works on one MNO's network only and is under the control of that MNO. Multiple repeater modules have been installed together to provide coverage for multiple MNOs. This approach addresses both the technical problems that cause interference. The number of mobile users on a train provides the economic justification. In-train repeaters are invariably professionally designed and installed with the full backing of the relevant $\mathrm{MNO}(\mathrm{s})$. However, in-car kits are also available for installation by consumers. These are essentially the same as the consumer in-building repeaters but with accessories more suited to a vehicle, e.g., magneticmount antennas and $12 \mathrm{~V}$ rather than mains power supply.

Repeaters mounted in vehicles will themselves be moving. The problem of a repeater operating close to a base station and raising the noise floor will occur from time to time as the vehicles pass base stations. For a repeater that operates across the whole band it will affect all networks in the band, including the wanted network. It would be possible to dynamically alter the repeater gain as the signal from the base station varies, but the implementation will need to be done in a way that avoids the power control problem.

\subsection{Wireless Access Points}

An access point is a device that creates a wireless local area network, or Wide Local Area Network or WLAN. It is mostly implemented in an office or large building. An access point is connected to a wired router, switch, or hub via an Ethernet cable. It projects a Wi-Fi signal to an elected area. For example, if it is prerequisite to enable $\mathrm{Wi}-\mathrm{Fi}$ access in a company's reception area but there's no Wi-Fi router within range, an access point near the front desk can be installed and run an Ethernet cable through the ceiling back to the server room.

For home Wi-Fi networks the range extenders are the best to use. But these access points are not proficient for contemporary businesses. As a result of that, these devices can support only an inadequate amount of end devices at once. The number is not more than twenty. The coverage area of a Wi-Fi router is extended by a range extender. But the available bandwidth is not increased by them. A range extender may cause a user to wait depending on the amount of concurrently connected devices.

On the contrary, access points are capable of providing services to over sixty concurrent connections at a stretch. The users get connected with the access point and can move independently without the concern of connectivity. As they will experience a seamless use while only getting connected once with an access point. Their connections will not be dropped. With the change of their locations their connectivity will be changed simultaneously with another access point near to the user. 
When there is both employees and guests connecting with desktops, laptops, mobile phones, and tablets, first twenty devices on a wireless network gets connected quickly. At 60 simultaneous connections each, access points give you the freedom to scale the number of devices supported on your network. But that's only one of the advantages of using these network enhancers.

Things to be considered are as following:

Access points which are of business grade can be installed anywhere where an Ethernet cable can be run. Power over Ethernet Plus, or PoE+ (a combination Ethernet and power cord), is also compatible with newer models of access points. There no extra source of power is required. It can take power from the Ethernet link.

Captive Portal and Access Control List (ACL) support is an additional standard feature. Guest access without compromising network security, as well as easily managing users within the Wi-Fi network can be limited by the administrator.

The selected access points must include a Clustering feature. Which is a single point from which the administrator can view, deploy, configure, and secure a Wi-Fi network as a single entity rather than a series of separate access point configurations

\subsection{Optical Fiber Network}

Optical fiber communication has transfigured the telecommunication industry. Within the data networking community, it has impacted widely its presence. By means of optical fiber cable, telecommunications links to be made over hugely greater distances with much lower levels of loss in the transmission medium. Much higher data rate has been accommodated now, enabled by optical fiber communications.

On account of these advantages, optical fiber communications systems are broadly active for applications ranging from major telecommunications backbone infrastructure to Ethernet systems, broadband distribution, and general data networking.

\subsubsection{Development of Fiber Optics}

There has been an ever increasing need to transmit more data even faster since the most primitive days of telecommunications. Single line wires were used primarily. These gave way to coaxial cables that enabled several channels transmitted over the identical cable. However, these systems were limited in bandwidth and optical systems were investigated.

After the first lasers were developed in the 1960s optical communications had become a possibility. when the first optical fibers with a sufficiently low loss for communications purposes were developed in the 1970s and then the next piece of the tangram fell into place. In the course of the late 1970s a substantial amount of research was instigated. This resulted in the installation of the first optical fiber telecommunications system. It loped in excess of a distance of $45 \mathrm{~km}$ and used a wavelength of $0.5 \mathrm{~mm}$ and had a data rate of just $45 \mathrm{Mbps}$.

Significant expansions have been made in the technology since then. The performance of the optical fiber has been improved to enable much greater distances to be achieved between repeaters. Data rates have been improved. Now the speeds can be achieved along through a fiber optic system exceed 10 Tbps.
It was alleged that the fiber optic cabling and technology would be exorbitantly expensive when the first fiber optic transmission systems were being technologically advanced. This has not been the circumstance and costs have fallen to the magnitude that fiber optics now provides the only worthwhile option for many telecommunications applications. Optical fiber is also used in many local area networks where speed is the most important constraint [11].

\subsubsection{Advantages of Fiber Optics}

There are a number of enthralling whys and wherefores that lead to the prevalent espousal of fiber optic cabling for telecommunications applications [12]:

- Considerable inferior echelons of signal attenuation

- Consenting more data to be carried fiber optic cabling be responsible for a much-sophisticated bandwidth

- Optical fiber cables are much nimbler than the coaxial cables that might else be used.

- Optical fiber does not writhe from vagrant intrusion pickup that ensues with coaxial cabling

\subsubsection{Fiber Optic Transmission System}

A number of diverse features consist of with any optical fiber data transmission scheme. There are three foremost components and an auxiliary one that is vivacious for realworld systems [12]:

- Transmitter (light source)

- Fiber optic cable

- Receiver (Detector)

- Optical repeater

The unalike rudiments of the system will diverge bestowing to the application. Systems used for inferior capacity links, possibly for local area networks will employ to some extent dissimilar methods and mechanisms to those used by network benefactors that be responsible for enormously high data rates over protracted distances. Nonetheless the rudimentary ideologies are equivalent to all the systems.

The transmitter of light source generates a light stream modulated to enable it to carry the data. Conventionally a pulse of light indicates a "1" and the absence of light indicates "0". This light is transmitted down a very thin fiber of glass or other apposite material to be obtainable at the receiver or detector. The detector transmutes the pulses of light into correspondent electrical pulses. The data can be transmitted as light over great distances in this manner.

\subsubsection{Transmitter}

A variety of semiconductor devices can be used even though the novel telecommunications optical fiber systems would have used large lasers. The most frequently used devices are light emitting diodes, LEDs, and semiconductor laser diodes.

The simplest transmitter device is the LED. The foremost advantage of it is that it is economical. This makes optical fibers epitome for low-cost applications where only petite runs are needed. Nevertheless, they have a number of hitches. Firstly, optical fibers proposition a very low level of efficacy. Merely about $1 \%$ of the input power cross the threshold of the optical fiber, and this means that high power drivers would be needed to provide adequate light to allow long distance transmissions to be completed. The other drawback of LEDs is that they crop what is labeled incoherent light that covers a pretty wide spectrum. Characteristically the spectral width is 
between 30 and $60 \mathrm{~nm}$ [12]. This means that any chromatic dispersion in the fiber will limit the bandwidth of the system.

LEDs are implemented predominantly in local-area-network applications. Because the data rates are archetypally in the range $10-100 \mathrm{Mb} / \mathrm{s}$ and transmission distances are a few kilometers in a view of their performance.

Lasers are used where higher levels of performance are obligatory, i.e., it is compulsory that the fiber optic link can maneuver over greater distances and with higher data rates, then. Although more costly, they offer some noteworthy advantages. In the first illustration fiber cables are competent to offer a higher output level, and furthermore to this the light output is directional and this empowers a much higher level of efficacy in the transfer of the light into the fiber optic cable. Stereotypically the coupling efficiency into a single mode fiber may be as high as $50 \%$. A supplementary benefit is that lasers have a very slender spectral bandwidth therefore the fact that lasers produce comprehensible light. This slender spectral width allows the lasers to conduct data at much higher rates because modal dispersion is less apparent. Another benefit is that semiconductor lasers can be curbed unswervingly in elevated frequencies on account of short recombination time for the carriers surrounded by the semiconductor material.

Laser diodes are time and again unswervingly restrained. This delivers a very guileless and in effect technique of transmitting the data onto the optical signal. This is attained by scheming contemporary smeared unswervingly to the device. This consecutively varies the light output from the laser. Nevertheless, for very high data rates or very longdistance links, it is more in effect to run the laser at a continuous output level (continuous wave). The light is then modulated using an exterior device. The benefit of using an external means of modulation is that it upsurges the maximum link distance because an effect known as laser squeak is eradicated. This chirp expands the spectrum of the light signal and this upsurges the chromatic dispersion in the fiber optic cable.

\subsubsection{Fiber Optic Cable}

Intrinsically a fiber optic cable entails of core, around which is another layer denoted to as the cladding. Exterior of this there is a protective outer coating.

The fiber optic cables function since their cladding has a refractive index that is to some extent lower than that of the core. This means that light fleeting down the core go through total internal reflection when it grasps the core / cladding boundary, and it is in so doing delimited in the interior the core of the optical fiber.

\subsubsection{Receivers}

Light itinerant alongside a fiber optic cable requires to be rehabilitated into an electrical signal so that it can be dealt with and the data that is passed can be mined. The factor that is at the core of the receiver is a photo-detector. This is customarily a semiconductor maneuver and may be a p-n junction, a p-i-n photo-diode or an avalanche photo-diode. Photo-transistors are not castoff as they do not have ample rapidity.

On one occasion of the optical signal from the fiber optic cable has been pragmatic to the photo-detector and rehabilitated into an electrical presentation it can be administered to make progress the data which can then be handed to its concluding terminus.
By and large data transmission of optical fibers are castoff for long aloofness telecommunications network links and for high speed local area networks. At this time fiber optics is not castoff for the conveyance of amenities to homes, even though this is a long tenure aim for many telecommunication companies. By expending optical fiber cabling, the obtainable bandwidth for novel amenities would be significantly higher and the likelihood of greater returns would upsurge. At this moment in time the price of this is not feasible, even if it is likely to transpire in the intermediate term.

\subsubsection{Repeaters and Amplifiers}

A thoroughgoing distance is there above which signals may be transferred over fiber optic cabling. This is restricted not only by the diminution of the cable, but also the distortion of the light signal beside the cable. Repeaters and amplifiers are used to overawed these properties and convey the signals over longer spaces.

For this reason, Opto-electric repeaters can be castoff. These devices transform the optical signal into an electrical presentation where it can be treated to confirm that the signal is not distorted and then transformed back into the optical presentation. It may then be transmitted laterally the subsequent phase of the fiber optic cable.

An unconventional slant is to consume an optical amplifier. These amplifiers unswervingly intensify the optical signal deprived of the need to transform the signal back into an electrical presentation. The amplifiers comprise of a length of fiber optic cable that is nobbled with a infrequent earth mineral named Erbium. The preserved fiber cable is then brightened or propelled with light of a shorter wavelength from another laser and this serves to amplify the signal that is being carried.

Amplifiers are far more widely used in view of the muchreduced cost of fiber optic amplifiers over repeaters. Amplifiers are used in fundamentally and most repeaters have been substituted, all new setting up these days.

\subsection{Vehicular Ad-hoc Network (VANET)}

In recent times Vehicular Ad hoc Networks are fetching one of the most auspicious research fields. VANETs which use vehicles as mobile nodes are a subclass of mobile ad hoc networks (MANETs) to offer communications amid neighboring vehicles and between vehicles and adjacent roadside apparatus [13]. But deceptively be at variance from other networks by their own physiognomies. More precisely, the nodes (vehicles) in VANETs are partial to road topology while moving. If the road statistics is obtainable, it is possible to foretell the forthcoming locus of a vehicle. Vehicles can afford noteworthy computing, communication, and sensing competences as well as providing incessant transmission power themselves to support these functions [14].

In VANETs, contributing vehicles are fortified with a set of wireless sensors and on-board units (OBUs) to permit for leeway of wireless communication between the vehicles and their environs. These devices make each vehicle function as packet sender, receiver and router which empower the vehicles send and receive communications to other vehicles or road side units (RSUs) within their grasp by means of wireless medium.

The progress of VANETs architecture diverges from expanse to expanse. In the CAR-2-X communication system which is trailed by the CAR-2-CAR communication consortium, the orientation architecture is a slight diverse. 
CAR-2-CAR communication consortium $(\mathrm{C} 2 \mathrm{C}-\mathrm{CC})$ is the most important pouring force for vehicular communication in Europe and published its "manifesto" in 2007. This system architecture encompasses three domains: in-vehicle, ad hoc, and infrastructure domain.

The in-vehicle domain is unruffled of an on-board unit (OBU) and one or multiple application units (AUs). The connections between them are usually wired and sometimes wireless. The ad hoc domain is composed of vehicles equipped with OBUs and roadside units (RSUs). An OBU can be seen as a mobile node of an ad hoc network and RSU is a static node likewise. An RSU can be connected to the Internet via the gateway; RSUs can communicate with each other directly or via multihop as well. There are two types of infrastructure domain access, RSUs and hot spots (HSs). OBUs may communicate with Internet via RSUs or HSs. In the absence of RSUs and HSs, OBUs can also communicate with each other by using cellular radio networks (GSM, GPRS, UMTS, WiMAX, and 4G) [14]

Communication types in VANETs can be categorized into four types. The category is closely related to VANETs components as described above [15].

In-vehicle communication, which is more and more compulsory and imperative in VANETs study, denotes to the in-vehicle domain. In-vehicle communication system can detect a vehicle's enactment and exclusively driver's exhaustion and lethargy, which is life-threatening for driver and public wellbeing.

Vehicle-to-vehicle (V2V) communication can provide a data interchange podium for the drivers to share information and warning messages, so as to swell driver succor.

Vehicle-to-road infrastructure (V2I) communication is one more beneficial research field in VANETs. V2I communication permits real-time traffic/weather updates for drivers and provides environmental recognizing and nursing.

Vehicle-to-broadband cloud (V2B) communication means that vehicles may communicate via wireless broadband apparatuses such as $3 \mathrm{G} / 4 \mathrm{G}$. As the broadband cloud may comprise more traffic information and monitoring data as well as infotainment, this type of communication will be useful for vigorous driver support and vehicle pursuing.

\subsection{Wireless Mesh Networks (WMN)}

As various wireless networks grow into the next cohort to be responsible for healthier services, a key technology, wireless mesh networks (WMNs), has materialized in recent times. In WMNs, nodes are encompassed of mesh routers and mesh clients. Each node functions not only as a host but also as a router, forwarding packets on behalf of other nodes that may not be within direct wireless transmission range of their destinations. A WMN is vigorously self-organized and selfconstituted, with the nodes in the network automatically founding and maintaining mesh connectivity among themselves (creating, in effect, an ad hoc network). This piece conveys many compensations to WMNs such as low up-front cost, easy network maintenance, robustness, and reliable service coverage.

Wireless mesh networks (WMNs) consist of mesh routers and mesh clients, where mesh routers have negligible suppleness and form the backbone of WMNs. They deliver network access for both mesh and conservative clients.
The incorporation of WMNs with other networks such as the Internet, cellular, IEEE 802.11, IEEE 802.15, IEEE 802.16, sensor networks, etc., can be proficient over and done with the gateway and bridging utilities in the mesh routers.

Mesh clients can be moreover stationary otherwise mobile, and can arrangement a client mesh network among themselves and with mesh routers.

WMNs are projected to determine the confines and to ominously expand the enactment of ad hoc networks, wireless local area networks (WLANs), wireless personal area networks (WPANs), and wireless metropolitan area networks (WMANs). They are enduring hasty evolution and stimulating frequent dispositions. WMNs will distribute wireless services for a large variety of applications in personal, local, campus, and metropolitan areas. Notwithstanding latest improvements in wireless mesh networking, many research defies linger in all protocol layers.

Deploying a WMN is not too difficult, because all the required components are already available in the form of ad hoc network routing protocols, IEEE 802.11 MAC protocol, wired equivalent privacy (WEP) security, etc.

WMNs consist of two types of nodes: mesh routers and mesh clients. Other than the routing capability for gateway/repeater functions as in a conventional wireless router, a wireless mesh router contains additional routing functions to support mesh networking.

To further improve the flexibility of mesh networking, a mesh router is usually equipped with multiple wireless interfaces built on either the same or different wireless access technologies. Compared with a conventional wireless router, a wireless mesh router can achieve the same coverage with much lower transmission power through multi-hop communications. Optionally, the medium access control (MAC) protocol in a mesh router is enhanced with better scalability in a multi-hop mesh environment.

mesh and conventional wireless routers are usually built based on a similar hardware platform. Mesh routers can be built based on dedicated computer systems (e.g., embedded systems) and look compact. They can also be built based on general-purpose computer systems (e.g., laptop/ desktop PC). Mesh clients also have necessary functions for mesh networking, and thus, can also work as a router.

The architecture of WMNs can be classified into three main groups based on the functionality of the nodes:

- Infrastructure/Backbone WMNs. The architecture is shown in Figure 12, where dash and solid lines indicate wireless and wired links, respectively. This type of WMNs includes mesh routers forming an infrastructure for clients that connect to them. The WMN infrastructure/ backbone can be built using various types of radio technologies, in addition to the mostly used IEEE 802.11 technologies. The mesh routers form a mesh of self-configuring, self-healing links among themselves. With gateway functionality, mesh routers can be connected to the Internet. This approach, also referred to as infrastructure meshing, provides backbone for conventional clients and enables integration of WMNs with existing wireless networks, through gateway/bridge functionalities in mesh routers. Conventional clients with Ethernet interface can be connected to mesh routers via Ethernet links. For conventional clients with the same radio technologies as mesh routers, they can directly communicate with mesh routers. 


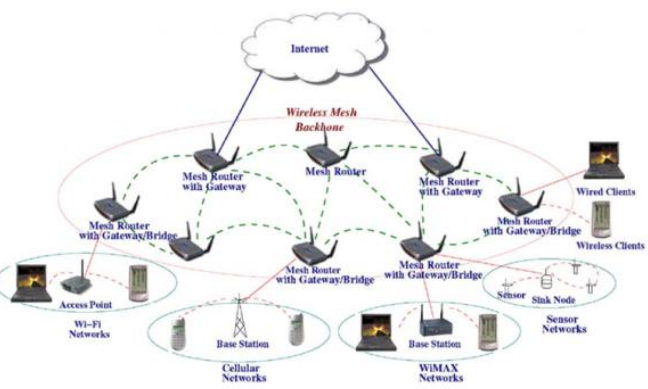

Fig 12: Infrastructure/Backbone WMNs

If different radio technologies are used, clients must communicate with the base stations that have Ethernet connections to mesh routers. Infrastructure/Backbone WMNs are the most commonly used type. For example, community and neighborhood networks can be built using infrastructure meshing. The mesh routers are placed on the roof of houses in a neighborhood, which serve as access points for users inside the homes and along the roads. Typically, two types of radios are used in the routers, i.e., for backbone communication and for user communication, respectively. The mesh backbone communication can be established using long-range communication techniques including directional antennas.

- Client WMNs. Client meshing provides peer-topper networks among client devices. In this type of architecture, client nodes constitute the actual network to perform routing and configuration functionalities as well as providing end-user applications to customers. Hence, a mesh router is not required for these types of networks. The basic architecture is shown in Figure 13.

In Client WMNs, a packet destined to a node in the network hops through multiple nodes to reach the destination. Client WMNs are usually formed using one type of radios on devices. Moreover, the requirements on end-user devices are increased when compared to infrastructure meshing, since, in Client WMNs, the end-users must perform additional functions such as routing and self-configuration.

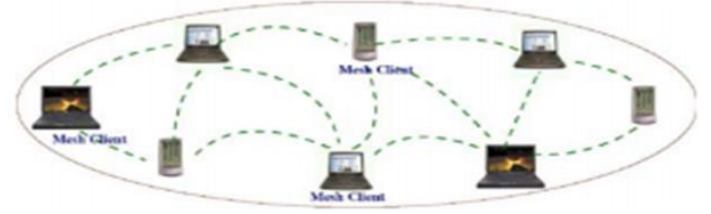

Fig 13: Client WMNs

- Hybrid WMNs. This architecture is the combination of infrastructure and client meshing as shown in Figure14. Mesh clients can access the network through mesh routers as well as directly meshing with other mesh clients. While the infrastructure provides connectivity to other networks such as the Internet, Wi-Fi, WiMAX, cellular, and sensor networks; the routing capabilities of clients provide improved connectivity and coverage inside the WMN. The hybrid architecture will be the most applicable case in our opinion.

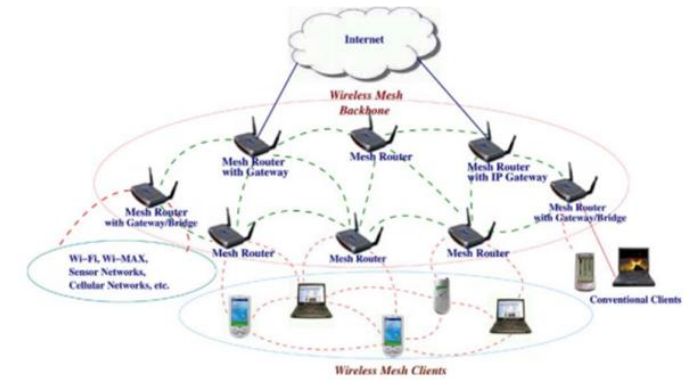

Fig 14: Hybrid WMNs

\subsubsection{Application Scenario of WMN}

Research and development of WMNs is motivated by several applications which clearly demonstrate the promising market while at the same time these applications cannot be supported directly by other wireless networks such as cellular networks, ad hoc networks, wireless sensor networks, standard IEEE 802.11, etc. Applications of WMNs are listed below:

Broadband home networking. Currently broadband home networking is realized through IEEE 802.11 WLANs. An obvious problem is the location of the access points. Without a site survey, a home (even a small one) usually has many dead zones without service coverage. Solutions based on site survey are expensive and not practical for home networking, while installation of multiple access points is also expensive and not convenient because of Ethernet wiring from access points to backhaul network access modem or hub. Moreover, communications between end nodes under two different access points have to go all the way back to the access hub. This is obviously not an efficient solution, especially for broadband networking.

Therefore, the communication between these nodes becomes much more flexible and more robust to network faults and link failures. Dead zones can be eliminated by adding mesh routers, changing locations of mesh routers, or automatically adjusting power levels of mesh routers. Communication within home networks can be realized through mesh networking without going back to the access hub all the time. Thus, network congestion due to backhaul access can be avoided. In this application, wireless mesh routers have no constraints on power consumptions and mobility. Thus, protocols proposed for mobile ad hoc networks [34] and wireless sensor networks $[8,9]$ are too cumbersome to achieve satisfactory performance in this application. On the other hand, Wi-Fis are not capable of supporting ad hoc multi-hop networking. As a consequence, WMNs are well-suited for broadband home networking.

- Community and neighborhood networking. In a community, the common architecture for network access is based on cable or DSL connected to the Internet, and the last-hop is wireless by connecting a wireless router to a cable or DSL modem. This type of network access has several drawbacks: - Even if the information must be shared within a community or neighborhood, all traffic must flow through Internet. This significantly reduces network resource utilization. - Large percentage of areas in between houses is not covered by wireless services. - An expensive but high bandwidth gateway between multiple homes or neighborhoods may not be shared and wireless services must be set up individually. As a result, network service costs may increase. - Only a single path may be available for one home to access the Internet or communicate with neighbors. 
- Enterprise networking. This can be a small network within an office or a medium-size network for all offices in an entire building, or a large-scale network among offices in multiple buildings. Currently, standard IEEE 802.11 wireless networks are widely used in various offices. However, these wireless networks are still isolated islands. Connections among them have to be achieved through wired Ethernet connections, which is the key reason for the high cost of enterprise networks. In addition, adding more backhaul access modems only increases capacity locally, but does not improve robustness to link failures, network congestion and other problems of the entire enterprise network. If the access points are replaced by mesh routers, as shown in given Figure, Ethernet wires can be eliminated. Multiple backhaul access modems can be shared by all nodes in the entire network, and thus, improve the robustness and resource utilization of enterprise networks. WMNs can grow easily as the size of enterprise expands. WMNs for enterprise networking are much more complicated than at home because more nodes and more complicated network topologies are involved. The service model of enterprise networking can be applied to many other public and commercial service networking scenarios such as airports, hotels, shopping malls, convention centers, sport centers, etc.

- Metropolitan area networks. WMNs in metropolitan area have several advantages. The physical-layer transmission rate of a node in WMNs is much higher than that in any cellular networks. For example, an IEEE $802.11 \mathrm{~g}$ node can transmit at a rate of $54 \%$ Mbps. Moreover, the communication between nodes in WMNs does not rely on a wired backbone. Compared to wired networks, e.g., cable or optical networks, wireless mesh MAN is an economic alternative to broadband networking, especially in underdeveloped regions. Wireless mesh MAN covers a potentially much larger area than home, enterprise, building, or community networks, as shown in given Figure. Thus, the requirement on the network scalability by wireless mesh MAN is much higher than that by other applications.

- Transportation systems. Instead of limiting IEEE 802.11 or 802.16 access to stations and stops, mesh networking technology can extend access into buses, ferries, and trains. Thus, convenient passenger information services, remote monitoring of in-vehicle security video, and driver communications can be supported. To enable such mesh networking for a transportation system, two key techniques are needed: the high-speed mobile backhaul from a vehicle (car, bus, or train) to the Internet and mobile mesh networks within the vehicle.

\section{PROPOSED METHOD}

\subsection{The Scenario}

In the ViNET infrastructure model, all transports staying on the roads will be considered as a node, as well as, the traffic and light posts will be used as nodes, which will play the role of the access points too. Though their characteristics and responsibilities will be a bit different than each other.

A scenario can be thought of, as, suppose you are walking through a pavement and suddenly you remembered that you had to pay a bill or book something online. There are no WiFi spots nearby you. You are out of time that you can return to your home and complete the task. Your mobile is out of balance and you are only with electronic money or no other source to recharge is available near you. You found that the activity is of very high priority and need to be done at that moment. So, you decide to pull out your mobile device supporting Wi-Fi. You search for available SSID. You found a government provided Wi-Fi signal, suppose - CITY_SSID. You get connected the internet simultaneously.

Another scenario is, in Dhaka city we spend a huge amount of time in traffic jam. It reduces our efficiency, puts adverse effect in the economy of the country as most of the time goes in roads while travelling. Sometimes it is quite costly to use cellular data and a person needs to completely depend upon the cellular connectivity provider. Now, to overcome this situation, with infrastructure, seamless connectivity to users can be provided. With traditional deployment style of access points, it will be required to install a lot of access points in the roads to cover up the entire city. But with the described infrastructure design, the costing can be reduced, though it is out of the scope. But it will surely be well enough to provide connectivity to a large of number of people. Also, the management will be easier.

\subsection{The Main Components}

The infrastructure will have the following components -
i) Controller
ii) Root Access Point
iii) Leaf Access Point
iv) Optical Fiber Link

\subsubsection{Controller}

A wireless LAN controller directs or regulates traffic on the wireless network. It is sold by WLAN controller vendors. It is a centralized Wi-Fi management device that manages all the access points in a campus.

At the back end, the data center (DC) will be responsible for making all the connectivity with the internet. At the DC there will be wireless access point controller located. These controllers will be responsible for controlling, monitoring all the Root access points. At the back end these controllers with play the most vital role. Also, there will be powerful and highly efficient routers and switches who can handle a large amount bandwidth.

The following points illustrates the reason to use a controller:

\subsubsection{Centralized Authentication}

No further distinct MAC address tables and apprising in each access point, controller provides for a centralized authentication contrivance over specific user name-password based Radius Server/ Active Directory/ LDAP Amalgamation, centralized MAC address filtering or certificate/ shared key centered authentication for all the clients from a principal site.

\subsubsection{Centralized Radio Management for All Access Points}

\subsection{Load Balancing}

The users are automatically shifted to adjacent access points if the load (number of users connecting) on one access point is high and the neighboring access point is lesser.

\subsection{Radio Balancing}

802.11 n enabled clients are connected to the 802.11 n radios, 802.11a enabled clients are connected to 802.11a radios; $802.11 \mathrm{~b} / \mathrm{g}$ enabled clients are connected to $802.11 \mathrm{~b} / \mathrm{g}$ radios in a multi-radio enabled access point.

\subsection{Inference Mitigation}

Adjacent Access Points are always maintained to operate in different non-overlapping channels by the controller so that 
there is no loss of packets due to interference in a dense wireless network.

\subsection{Fail Over}

Clients are automatically shifted to neighboring access points if any access point suddenly fails, thereby introducing redundancy in the network.

\subsubsection{RF Visualization}

Another advantage of today's centralized wireless networks are the visualization capabilities of the Controller. Once the Floor plan of the campus is integrated with the controller, the coverage pattern, signal strength, users associated in each access point and various parameters can be viewed LIVE over a PC monitor (through a web-based application) sitting in a central location. This makes monitoring and troubleshooting of networks very easy. You can also locate any active Wireless client in the network map by just typing its MAC ID in the software.

\subsubsection{Network Access Control Based on User Identity}

With today's centralized Controller based Wireless networks, wireless users can be further segregated in to sub-groups and each group can be given separate network access policies. For example, all the wireless users accessing the network from the finance department can be given SAP/ERP access while the sales department can be denied the same. Internet access for the junior management staff can be blocked and guests can be given temporary internet access without giving access to internal network. IT department and senior management can be given full unrestricted access to the network resources. Certain laptops/ wireless clients can even be blocked network access if they do not have the latest versions of the anti-virus/ OS patch running on their systems.

\subsubsection{Security}

After authentication, all the wireless packets are encrypted end to end using 128-bit encryption technology making it difficult for any casual intruders to get in to your network.

Wireless Intrusion Detection/ Prevention Systems (Where dedicated access points can act as scanners for wireless threats) can identify and block a whole range of wireless attacks like:

1. Ad-hoc network

2. Misassociation of AP/Client to other network access points

3. Rogue Access Points detection and prevention

4. Multiple futile attempts to connect to the wireless network

5. Honey pot attacks/ Man-In-The-Middle Attacks

6. Denial of Service Attacks etc.

Branch offices and remote offices are also protected as the controller can form a Secure VPN tunnel between the HO and branch locations. Rogue Access Points and Laptop's can be even located using location visualizers.

\subsubsection{Quality of Service Through Traffic Prioritization}

A centralized controller based wireless infrastructure can identify and differentiate between different types data packets and prioritize the critical traffic on the wireless network infrastructure - This is crucial for real time wireless traffic like voice, video etc.

\subsubsection{Root Access Point}

Root access points will be attached with the traffic light post at the road intersections. These root access points will have direct connection with the controller located at the DC through optical fiber connectivity.

All Root APs will be broadcasting a SSID, ex- CITY_WIFI. It will only be using $5 \mathrm{GHz}$ band to providing connectivity. It will not be responsible for client association. It will be connected with the Leaf APs, while the Leaf AP will be responsible for client association.

Centrally from the DC, these Root APs can be monitored and controlled.

In VANET environment there is a component named, RSU or road side unit. This unit has the functionality like the root AP. But in ViNET, access points will be the only infrastructure element that will be static. Also, in VANET connection is wireless with the central station or base station but in ViNET with the DC connectivity is wired, optical fiber connectivity.

Root Access Points must have the following criteria:

Default operating mode, which serves clients on both $2.4 \mathrm{GHz}$ and $5 \mathrm{GHz}$

- Dual 5-GHz mode, which serves clients on both $5-\mathrm{GHz}$ radios

- Wireless security monitoring, which scans both $2.4 \mathrm{GHz}$ and

$5 \mathrm{GHz}$ for security threats while also serving $5-\mathrm{GHz}$ clients

\subsubsection{Leaf Access Point}

Leaf access points will be attached with the vehicles running on the roads. That means all the vehicles running on the roads will be considered as an access point. These access points will do both range extension and client association. The Leaf APs will be WDS or Wireless Distribution System enabled. WDS is a technology that enables an access point to work both as an extender and client association.

Leaf APs will be supporting $2.4 \mathrm{GHz}$ and $5 \mathrm{GHz}$ band. A Leaf AP will be always looking for a signal, or SSID that is broadcasted all around the city as described previously. When a Leaf AP comes into the signal range of Root AP SSID, ex$500 \mathrm{~m}$, which is the standard range of a Root AP, will be capturing the SSID. When leaf AP will get connected with that SSID, as it will be WDS enabled, so the leaf AP will be rebroadcasting the SSID with both of its antennas. The 2.4 $\mathrm{GHz}$ band will be responsible for the client association and 5 $\mathrm{GHz}$ band will do the task of link establishment with the Root $\mathrm{AP}$ and extension so that, other leaf AP can get connected with it, if that AP is out of the range of the root AP.

Leaf AP will be prioritizing the root AP signal first. If root $\mathrm{AP}$ signal is not found then the leaf AP will look for signals from its nearby leaf AP. It will get connected with leaf AP with highest signal strength.

Leaf AP will be responsible for client association and signal extension.

\subsubsection{Optical Fiber Link}

Optical fiber link provides better link stability than any other medium. This will be used for the connectivity of the root APs with the main DC, where the controllers will be located. Link redundancy will be provided so that, if a link fails then other link might get on line. 


\subsection{Network Design}

The entire Dhaka city will be under same network. For better maintainability and quality of services city will be split into parts. But altogether the city will be considered as a single network. So that with the change of client connectivity with different vehicles do not put any impact in the link establishment.

\subsection{IP Distribution}

As there will be numerous amounts of access points as well as clients, IPv4 will not be enough to accommodate this. For that reason, IPv6 will be used. This is will be well enough for the network as it will generate tremendous number of IPs for the entire communication process.

IP version 6 (IPv6) is a new version of the Internet Protocol, designed as the successor to IP version 4 (IPv4). IPv6 increases the IP address size from 32 bits to 128 bits, to support more levels of addressing hierarchy, a much greater number of addressable nodes, and simpler auto-configuration of addresses. The scalability of multicast routing is improved by adding a "scope" field to multicast addresses. And a new type of address called an "anycast address" is defined, used to send a packet to any one of a group of nodes.

\subsection{Communication Process Between Root and Leaf AP}

Link range for a root AP is $500 \mathrm{~m}$ (approx.). While leaf AP will cover an area of $120 \mathrm{~m}$. When a leaf AP will be in the range of a root $\mathrm{AP}$, it will get connected with the root $\mathrm{AP}$. But if a leaf node is out of the range of the root them it will search for signals from other nearby AP who have been already connected with the root $\mathrm{AP}$ and extended its range. The requesting leaf AP will get connected with the leaf AP providing the strongest signal.

For clients, they will get connected with the nearest leaf AP. If the nearest leaf AP has already reached its maximum client allocation, then it will look for another one with good signal strength.

\subsection{Coverage Area}

Average coverage area of these devices is as listed in the below table:

Table 1: Average Coverage Area with Maximum Output Power

\begin{tabular}{|c|c|}
\hline Coverage Area (sq m) & $\begin{array}{c}\text { Maximum Output Power } \\
\text { (dBm) }\end{array}$ \\
\hline $\mathbf{1 5 0 - 2 5 0}$ & -17 \\
\hline $\mathbf{5 0 0}$ & -20 \\
\hline $\mathbf{1 0 0 0 - 1 5 0 0}$ & -21 \\
\hline $\mathbf{2 5 0 0 - 3 0 0 0}$ & -23 \\
\hline $\mathbf{5 0 0 0}$ & -27 \\
\hline
\end{tabular}

Maximum Gain is listed in the below table:

Table 2: Maximum coverage area with maximum gain

\begin{tabular}{|c|c|}
\hline $\begin{array}{c}\text { Maximum Coverage Area } \\
\text { (sq m simple) }\end{array}$ & $\begin{array}{c}\text { Typical Maximum Gain } \\
\text { (dBm) }\end{array}$ \\
\hline $\mathbf{1 5 0 - 5 0 0}$ & $55-60$ \\
\hline $\mathbf{1 0 0 0 - 2 5 0 0}$ & $60-65$ \\
\hline Over 2500 & $65-75$ \\
\hline
\end{tabular}

\section{SIMULATION AND ANALYSIS}

Simulation has been run in Arena Simulation tool with the processes that are going to take place in the infrastructure model's components.

There were 500 vehicles or nodes or access points been taken. The simulation was run for 11 minutes. The final outcome along with the simulation flowchart is given below:

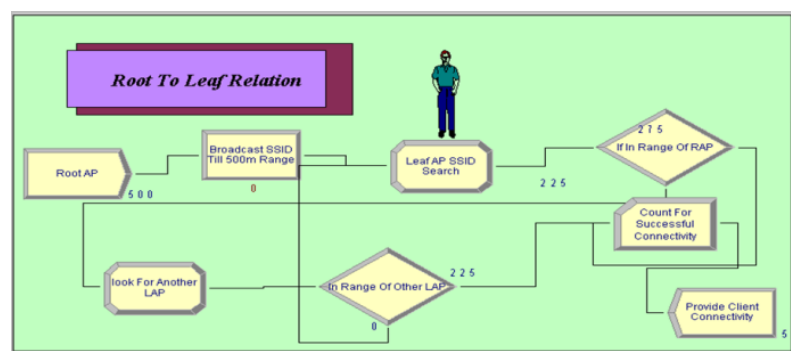

Fig 15: Root AP to Leaf AP Relation

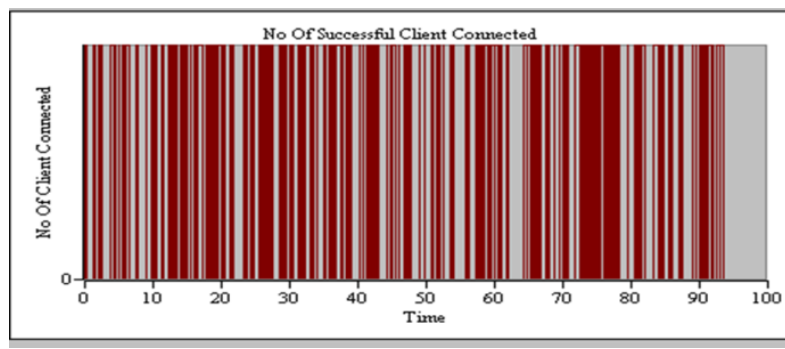

Fig 16: No of Successful Client Connectivity

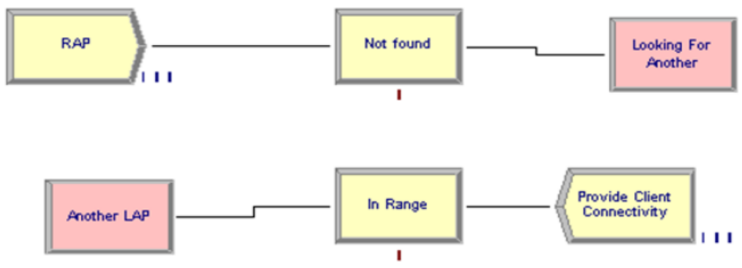

Fig 17: Leaf AP to Leaf AP Relation

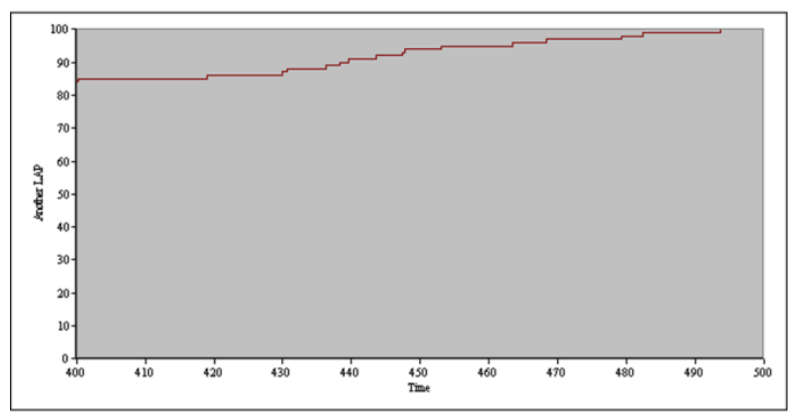

Fig 18: Time to get connected with another LAP

In Figure 15 and Figure 17 root AP to leaf AP connectivity process and leaf AP to leaf AP connectivity analysis is shown. From Figure 17 it is shown that the ViNET infrastructure can easily accommodate a huge number of clients in the re

\section{CONCLUSION AND FUTURE WORK}

The main objective of this research was to find a better way to utilize one of the greatest problems of Dhaka city in a better way. Existing technologies in our country as well as a very interesting and hot topic in the modern world, VANET, its concept is also been implemented in the infrastructure model. 
With the simulation driven results it is shown that the proposed infrastructure is well capable of providing connectivity to the city residents. The security and cost issues were not considered in this research. These issues are the scope of the future works. Also, by implementing this infrastructure the government can ensure seamless connectivity to city residents and they can utilize their time while stuck in a traffic jam or going through a pavement beside a road. Only Wi-Fi supported devices is the requirement for the users. Also, as in VANET for vehicle-tovehicle communication different medium named DSRC (Direct Short-range Communication) is used. In case of ViNET, Wi-Fi is the only medium that is used to complete the communication process. Also, government can use this service for other purposes, by creating a portal, where important road or emergency service-related information can be achieved. This infrastructure will need a great amount of manpower. It will create new job roles also for network engineers. Efficient routing algorithms should be invented to make sure the fastest data connectivity in the network as the topology is changeable rapidly. With the help of this ViNET infrastructure access to information to everyone in the urban area Bangladesh can be achieved.

\section{REFERENCES}

[1] Vehicular Ad Hoc Networks: Architecture, Research Issues, Methodologies, Challenges, and Trends

[2] Network map design for QoS routing on WAN Tomokazu Masuda, Keita Kawano, Hideaki Tanioka, Kazuhiko Kinoshita, Tetsuya Takine, Koso Murakami Fifth Asia-Pacific Conference on... and Fourth Optoelectronics and Communications Conference on Communications, 1, 196-199, 1999

[3] Feasibility Study of Mesh Networks for All-Wireless Offices,

[4] Wireless mesh networks: WMN overview, WMN architecture; Mojtaba Seyedzadegan, Mohamed Othman, Borhanuddin Mohd Ali, Shamala Subramaniam International conference on communication engineering and networks IPCSIT 19, 2, 2011

[5] Serval mesh software-wifi multi model management
Paul Gardner-Stephen, Swapna Palaniswamy; Proceedings of the 1st International Conference on Wireless Technologies for Humanitarian Relief, 71-77, 2011https://www.ietf.org/rfc/rfc3561.txt

[6] RFC3561: Ad hoc on-demand distance vector (AODV) routing;Charles Perkins, Elizabeth Belding-Royer, Samir Das; RFC Editor, 2003

[7] Wireless condition monitoring system for large vessels: Development and Application ; Min-chan Shim, Bo-suk Yang, Young-mo Kong, Won-cheol Kim; Engineering Asset Lifecycle Management, 637-643, 2010

[8] Tecnologías wireless y movilidad en IPv4/IPv6 Luis Armando Marrone, Andrés Barbieri, Matías Robles Editorial de la Universidad Nacional de La Plata (EDULP), 2011

[9] Configuring the Wireless Distribution System (WDS) Website: http://support.dlink.com/emulators /dwl2210ap/help/wds.help.html, date accessed 16/6/13

[10] Review of the present status of optical fiber sensor; School of Electrical Engineering, Seoul National University, Kwanak-Gu Shinlim-Dong, Seoul 151-744, South Korea

[11] Fundamentals of fibre optics in telecommunication and sensor systems;Bishnu P Pal; Bohem press, 1992

[12] Converged wireline and wireless signal transport over optical fibre access links Idelfonso Tafur Monroy, Kamau Prince, Alexey Osadchiy, Neil Guerrero González, Antonio Caballero, Darko Zibar, Tim Gibbon, Xianbin Yu, Jesper Bevensee Jensen 2009 14th OptoElectronics and Communications Conference, 1-2, 2009

[13] M. Sivasakthi and S. Suresh, "Research on vehicular ad hoc networks (VANETs): an overview," Journal of Applied Sciences and Engineering Research, vol. 2, no. 1, pp. 23-27, 2013.

[14] H. Moustafa and Y. Zhang, Vehicular Networks: Techniques, Standards, and Applications, CRC Press, Boca Raton, Fla, USA, 2009.

[15] M. Faezipour, M. Nourani, A. Saeed, and S. Addepalli, "Progress and challenges in intelligent vehicle area networks," Communications of the ACM, vol. 55, no. 2, pp.90-100,2012. 